\title{
Reducing Barriers to Bystander Intervention: Piloting a Wintersession Program
}

\author{
Truelian Lee, EMT-B
}

POSTER PRESENTATION ABSTRACT | PROGRAM DEVELOPMENT \& EVALUATION CATEGORY

Introduction: Occurring during the last week of winter recess, Harvard University's Wintersession offers enrichment programming. Similar programs exist in various other colleges, including Brown University, Wellesley College, and Ithaca College. Harvard Crimson EMS saw Wintersession as an opportunity to reach students who are too busy during the school year to learn bystander intervention skills. Consequently, the organization piloted a series of Wintersession workshops for the first time this year.

Program Development \& Implementation: Over the period of a month, Crimson EMS planned workshops that occurred from 10 AM-3 PM on January 22-25, 2019. Students earned national certifications in AHA CPR Heartsaver/First Aid and Stop the Bleed. Crimson EMS instructors used a curriculum based on prior outreach efforts. Lectures were conducted with PowerPoint presentations and skills practice with manikins or with partners and instructors. The total budget for the program was $\$ 450$. Registration was capped at 15 students based on the number of available instructors. Crimson EMS primarily publicized the program through social media.

Program Evaluation: 14 students attended. Crimson EMS solicited free response feedback as well as Likert scale ratings with 1 representing "poor" and 5 "excellent" to evaluate the program. At least $40 \%$ of participants had no prior experience to the skills taught. On average, participants rated the overall program a $4.2(\mathrm{SD}=0.8)$, with the ratings for specific workshops ranging from $3.9(\mathrm{SD}=1.4)$ for common collegiate EMS cases to $4.8(\mathrm{SD}=0.3)$ for cardiopulmonary resuscitation. Participants gave the lectures a $3.9(\mathrm{SD}=1.1)$ and the skills practice sections a $5.0(\mathrm{SD}=0.0)$.

Discussion/Conclusions: Based on participant responses, we plan to include additional skills training in future workshops and publicize the program through more platforms and for a longer period of time. This Wintersession represents a new avenue for Crimson EMS to promote collegiate EMS and educate community members about important, life-saving skills to reduce the barrier to bystander intervention.

Author Affiliations: Crimson EMS, Harvard University, Cambridge, MA, USA Address for Correspondence: Truelian Lee, EMT-B.

Email: truelianlee@college@harvard.edu

Conflicts of Interest/Funding Sources: By the JCEMS Submission Declaration Form, all authors are required to disclose all potential conflicts of interest and funding sources. The authors declared that they have no conflicts of interest. The authors declared that they received funding from Harvard University Undergraduate Council. Ethical Compliance: The authors attest that the research associated with this abstract was conducted in accordance with the JCEMS Ethics Guidelines.

Submission History: Received January 29, 2019; accepted for presentation and publication February 8, 2019.

Poster Presentation: This abstract was presented as a poster at the Academic Poster Session of the $26^{\text {th }}$ Annual Conference of the National Collegiate Emergency Medical Services Foundation; February 23, 2019; Pittsburgh, PA, USA.

Published Online: December 31, 2019

Published in Print: December 31, 2019 (Volume 2: Supplemental 1)

Reviewer Information: In accordance with JCEMS editorial policy, poster presentation abstracts undergo double-blind peer-review by at least two reviewers (JCEMS Editorial Board members and/or independent reviewers) prior to acceptance for presentation and publication. JCEMS thanks the anonymous reviewers who contributed to the review of this work.
Copyright: $\odot 2019$ Lee. This is an open access abstract distributed under the terms of the Creative Commons Attribution 4.0 International (CC BY 4.0) License, which permits unrestricted use, distribution, and reproduction in any medium, provided the original author and source are credited. The full license is available at: https://creativecommons.org/licenses/by/4.0/

Electronic Link: https://doi.org/10.30542/JCEMS.2019.02.S1.06 